\title{
Spectrum and hematological profile of hereditary anemia in North Indians: SGPGI experience
}

\author{
Poonam Tripathi, Ravindra Kumar, Sarita Agarwal* \\ Department of Medical Genetics, Sanjay Gandhi Post Graduate Institute of Medical Sciences (SGPGIMS), Lucknow, India.
}

\begin{abstract}
Summary Thalassemia and other hemoglobinopathies together with red cell enzymopathies are a common cause of anemia, which can be prevented by population screening and genetic counseling. This study was designed to screen the anemic patients for thalassemia, structural hemoglobin variants and red cell enzymopathies. A total of 17047 cases were evaluated from 2009 to 2018 for thalassemia, hemoglobin variants, glucose 6 phosphate dehydrogenase deficiency, pyruvate kinase deficiency and hereditary spherocytosis. Patients' records were entered in a Microsoft excel sheet and a spectrum of disorders was evaluated. Year wise spectrum was also analyzed to see the difference in incidence at different time periods. Incidence of beta thalassemia trait and thalassemia major was found in $\mathbf{1 1 . 0 \%}$ and $\mathbf{3 . 4 \%}$ respectively, whereas other hemoglobinopathies were observed in $3.2 \%$ of the cases. G6PD deficient cases were $0.2 \%$ and $0.4 \%$ had hereditary spherocytosis. No significant difference was observed in incidence of thalassemia and other hemoglobinopathies at different time points. This study provided a health burden and detailed spectrum and prevalence of hemoglobinopathies in North Indians high risk population which contribute toward the development of prevention strategies for better management of hemoglobinopathies. In view of high incidence of thalassemia a routine hematological screening at a primary health center may be introduced as a prospective premarital screening under a thalassemia control program. Moreover rapid and easy quantification of hemoglobin variants (Hb variants) make Cation exchange - High Performance Liquid Chromatography (CE-HPLC) a suitable diagnostic test for the routine investigation of genetic causes of anemia.
\end{abstract}

Keywords: Thalassemia, Hb variants, G6PD deficiency, Premarital Screening, HPLC

\section{Introduction}

The inherited disorders of hemoglobin synthesis are the most common monogenic disorders worldwide. Hemoglobinopathies are a group of inherited disorders of hemoglobin synthesis and a major cause of anemia. Hemoglobinopathies responsible for hemolytic anemia may be divided into two groups. The first one corresponds to thalassemia and the second to the presence of structurally abnormal hemoglobin. Though, there are many variants of thalassemia, $\beta$-thalassemia is the most common variant found in the Indian subcontinent. Since thalassemia is difficult to cure, it

\footnotetext{
*Address correspondence to:

Dr. Sarita Agarwal, Department of Genetics, Sanjay Gandhi Post Graduate Institute of Medical Sciences (SGPGIMS), Lucknow 226014, India.

E-mail: saritasgpgi@gmail.com
}

becomes a priority to prevent this disorder. The clinical spectrum of these disorders varies from asymptomatic conditions (beta-thalassemia minor) to serious disorders such as thalassemia major that require regular blood transfusions and extensive medical care. The only cure for affected children is bone marrow transplantation, which is expensive, risky and difficult to perform with discouraging success rates. The other treatment to sustain life is regular blood transfusion with iron chelation but this is very painful. As anemia is considered a major public health problem in India, so early detection of anemia in these disorders allows timely intervention and prevention from serious consequences.

The most common effective approach for developing countries like India is preventing disorders associated with $\beta$-globin gene cluster and major efforts need to be directed at applying a simple and well defined strategy to control these disorders by carrier detection, genetic 
counseling and prenatal diagnosis (1). However, accurate and timely detection of various known and unknown $\mathrm{Hb}$ variants can prevent occurrence of serious $\mathrm{Hb}$ disorders such as thalassemia major in newborns.

Mohanty et al. in 2013 (2) conducted a multicenter study and after analyzing 56,780 samples from 6 different centers; they reported overall a $2.78 \%$ prevalence of thalassemia trait in the studied cohort. They highlight that the prevalence of $\beta$-thalassemia varied from 0 to $10.5 \%$ among the different caste/ethnic groups of India (2).

Since there is lot of complexity in the Indian population structures and due to high demand for resources; large migration of communities occured in the past from one geographical area to another, and therefore there is a need to evaluate the prevalence of hemoglobin disorders from time to time.

The present study was carried out to determine the prevalence of hemoglobinopathies and $\beta$-thalassemia among the North Indian populations from 2009-2018. In addition, our study also aimed to identify rare/unknown Hemoglobin variants by molecular analysis to provide accurate diagnosis and genetic counseling.

\section{Materials and Methods}

This cross-sectional descriptive study was conducted in Department of Medical Genetics, Sanjay Gandhi Postgraduate Institute of Medical Sciences, Lucknow from 2009 to 2018. Individuals with suspected risk of thalassemia and other hemoglobinopathies, with known family history, premarital, preconception (postmarital screening before planning child birth) or antenatal subjects who apparently did not have any suspicion of risk of carrier state and individuals coming voluntarily for screening were included.

To evaluate the cause of anemia a battery of tests related to hematological and biochemical parameters such as Glucose-6-phosphate dehydrogenase deficiency (G6PD), Pyruvate kinase Enzyme Deficiency, Hereditary Spherocytosis and Iron deficiency Anemia (IDA) were carried out.

Four ml blood sample was collected from each individual in ethylene diamine tetra acetic acid (EDTA) and plain vials. Red Cell indices analysis was carried out on a Sysmex 21 automated blood cell counter for red cell indices.

Serum Iron and total iron binding capacities (TIBC) was estimated by the Ferrozine method (International Council for Standardization in Hematology 1990) and using IRON \& TIBC kit (Crest Biosystems, India) on the same day of blood collection.

The samples were stored at $4^{\circ} \mathrm{C}$ and further analyzed in batches for CE-HPLC (Cation exchange - High Performance Liquid Chromatography). CE-HPLC was performed with each blood sample on a BIO-RAD Variant II using beta thalassemia short program pack.
DCIP test was performed for G6PD deficiency. Dichloro phenol indophenols decolorizing test (DCIP Bernstein test) is a screening dye test for G6PD deficiency diagnosis. Nicotinamide adenine dinucleotide phosphate (NADP) reduces the dye DCIP into a colorless state (DCIPH) through the action of G6PD. The rate and the degree of this decolorization are proportional to the G6PD activity in RBCs. Phenazonemethosulphate (PMS) is used as an electron carrier between NADPH and 2-6 DCIP in the test. $20 \mu \mathrm{L}$ of whole blood followed by $400 \mu \mathrm{L}$ of DCIP dye solution was mixed in a test tube containg $50 \mu \mathrm{L}$ of PMS, $25 \mu \mathrm{L}$ of G6P and $25 \mu \mathrm{L}$ of NADP and $1 \mathrm{~mL}$ of distilled water and overlaid with liquid paraffin incubate at $37^{\circ} \mathrm{C}$ for $10 \mathrm{~min}$. The mixture color changes from blue to red in presence of G6PD deficiency within $10 \mathrm{~min}$.

Pyruvate kinase (PK) deficiency is due to defects in pyruvate kinase enzyme expression or activity. Pyruvate kinase enzyme catalyzes the last step of glycolysis, the transfer of a phosphate group from phospho(enol) pyruvate (PEP) to ADP, yielding one molecule of pyruvate and one molecule of adenosine triphosphate (ATP). In the pyruvate kinase deficiency test, PEP and ADP were catalyzed by PK to generate pyruvate and ATP. The generated pyruvate is oxidized to produce fluorescence (at $340 \mathrm{~nm}$ ). The $1000 \mu \mathrm{L}$ reaction mixture in a tube contained $25 \mathrm{mM}$ potassium phosphate buffer pH $7.450 \mu \mathrm{L}, 8 \mathrm{mM}$ magnesium sulfate $100 \mu \mathrm{L}, 0.15 \mathrm{mM}$ NADH $100 \mu \mathrm{L}, 0.15 \mathrm{mM}$ phosphoenolpyruvate $30 \mu \mathrm{L}$, $0.3 \mathrm{mM}$ ADP $100 \mu \mathrm{L}$, and water $620 \mu \mathrm{L}$. RBC solution was prepared using packed red blood cells suspended in $20 \%$ normal saline. $200 \mu \mathrm{L}$ reaction mixture was mixed with $20 \mu \mathrm{L}$ RBC solution in a tube and the mixed solution was spotted on Whatman no 1 filter paper at zero time. Tubes were placed in water bath at $37^{\circ} \mathrm{C}$ for 10 minutes and again a drop from the mixture was spotted on filter paper after 20 and 30 minutes. In a normal sample disappearance was seen with in $30 \mathrm{~min}$ but in pyruvate deficient samples fluorescence takes a longer time to disappear. Since the increase in color or fluorescence intensity is proportional to the increase in pyruvate amount, the PK activity can be accurately measured.

Osmotic fragility is a test to measure the resistance to hemolysis of RBCs exposed to a series of hypotonic solutions. This test is performed to detect thalassemias, red cell membrane disorders and hereditary spherocytosis. In the osmotic fragility test, whole blood is added to varying concentrations of buffered sodium chloride solution and allowed to incubate at room temperature. The amount of hemolysis in each saline concentration is then determined by reading the supernatants on a spectrophotometer at $540 \mathrm{~nm}$. The results of the test may then be graphed, with the percent hemolysis plotted on the vertical axis and the sodium chloride concentration on horizontal axis.

The flow diagram for sample processing is depicted 
in Figure 1.

Statistical analysis: Data is presented as number and percentages for discrete variables and as mean \pm standard deviation for the continuous variables. The chi square test for trend analysis was done to see the pattern of change of prevalence of different studied causes of hemolytic anemia.

\section{Results}

Out of 17,047 cases, 3,379 cases were found with normal red cell indices (Table 1). High value of mean corpuscular volume $(\mathrm{MCV}>100 \mathrm{fl})$ and mean corpuscular hemoglobin $(\mathrm{MCH}>36 \mathrm{pg}$ ) were found in 211 cases. These 211 cases were found to be Vitamin B12 deficient and excluded for further study. Low Mean corpuscular volume $(<76 \mathrm{fl})$ and Low mean corpuscular hemoglobin $(<26 \mathrm{pg})$ was found in 13,457 cases. Out of these 13,457 samples, 10,201 cases were identified as iron deficiency anemia on the basis of their Serum Iron and Total Iron Binding Capacity (TIBC) levels. G6PD deficiency was found in 37 cases where as hereditary spherocytosis in 75 cases was noted (Table 1). We did not find any case of pyruvate kinase deficiency during the study period.

The remaining samples of 2,607 individuals were further analyzed for thalassemia and other hemoglobinopathies. After doing HPLC 1,764 cases were identified with abnormal hemoglobin variants.

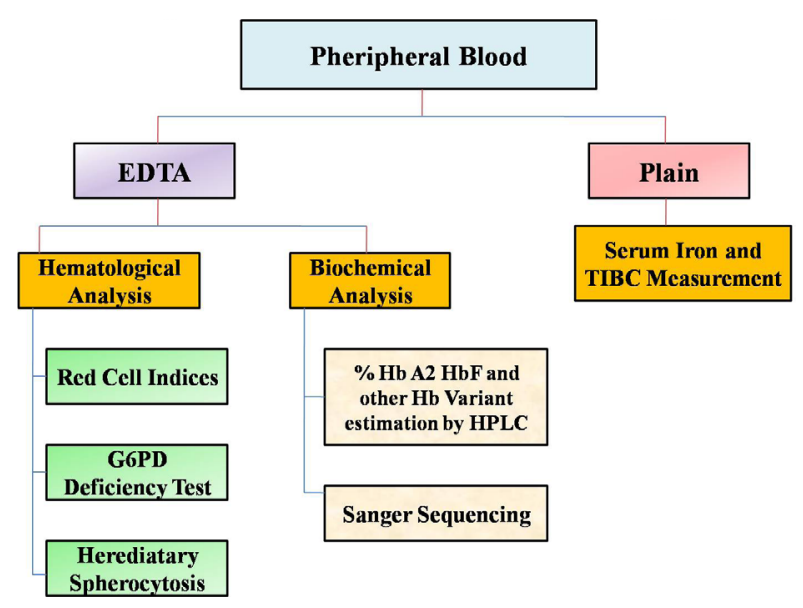

Figure 1. Flow chart showing detail methodology.
These 1,764 cases were further sub characterized on the basis of their clinical and biochemical evaluation like thalassemia major (579) and thalassemia trait $(1,185)$.

Hemoglobin variant disorders found in the present study were $\mathrm{HbE}$ trait (204), HbE disease (68), E $\beta$ thalassemia (51), $\mathrm{HbS}$ trait (187), HbS disease (136), $\mathrm{S} \beta$ thalassemia (85), $\mathrm{HbD}$ trait (85), $\mathrm{HbS} / \mathrm{HbE}$ Double heterozygous state (17) and $\mathrm{HbS} / \mathrm{HbD}$ double heterozygous state (34).

Red cell indices were carried out in all samples as this is a primary screening step to find out the cause of anemia. Since thalassemia is a disorder of anemia, hemoglobin values were found reduced in thalassemia along with the microcytic and hypochromic RBCs. Low MCV and low $\mathrm{MCH}$ values were recorded in cases as compared to normal. The mean hemoglobin levels, $\mathrm{MCV}$ and $\mathrm{MCH}$ values of all thalassemia groups are shown in Table 2. $\mathrm{MCV}$ and $\mathrm{MCH}$ values were lowest in EE group.

HPLC analysis was carried out in subjects with low $\mathrm{MCV}$ and $\mathrm{MCH}$ and without iron deficiency to find out the status of thalassemia and other hemoglobinopathies. Comparison of mean percentages of various hemoglobins in each thalassemia group are shown in Table 3.

Year wise comparison was done to see whether the prevalence of thalassemia was increasing or decreasing by time and we observed that there was no trend in prevalence of thalassemia and other hemoglobinopathies across different time intervals $(p=0.826)$ (Table 4).

In this study, molecular characterization of beta globin gene sequencing identified a rare HB Köln (HBB:c.295 G>A) and a novel single nucleotide deletion at codon 79 (HBB:c240_240delC) (3).

Defining genetic changes will be helpful to explain the clinical findings of the patient and for providing accurate diagnosis and genetic counseling. Therefore, it is important to report rare nucleotide changes in hemoglobin diseases and molecular analysis by Sanger sequencing so that prevalence of various hemoglobin variant can be monitored carefully.

\section{Discussion}

The present study is an expansion of our previous study published in Prenatal diagnosis (1) in which we have done screening of thalassemia and other hemoglobin disorders in extended family members of thalassemia

Table 1. Incidence of different disorders causing anemia in studied cohort

\begin{tabular}{|c|c|c|c|}
\hline Diagnosis & Male & Female & Total \\
\hline Normal & $2,273(31 \%)$ & $1,643(16.9 \%)$ & $3,916(22.9 \%)$ \\
\hline Megaloblastic anemia & $94(1.3 \%)$ & $117(1.2 \%)$ & $211(1.2 \%)$ \\
\hline Iron deficiency anemia & $3,555(48.5 \%)$ & $6,646(68.4 \%)$ & $10,201(59.8 \%)$ \\
\hline Hereditary Spherocytosis & $37(0.5 \%)$ & $38(0.4 \%)$ & $75(0.4 \%)$ \\
\hline G6PD deficient & $37(0.5 \%)$ & 0 & $37(0.2 \%)$ \\
\hline Thalassemia and Hemoglobin variants & $1,334(18.2 \%)$ & $1,273(13.1 \%)$ & $2,607(15.2 \%)$ \\
\hline Total & $7,330(43 \%)$ & $9,717(57 \%)$ & 17,047 \\
\hline
\end{tabular}

() number in parenthesis represents percentages. 
patients, couples coming for premarital screening and college going students. In that study we concluded that a cost effective approach for preventing birth of a thalassemia child is to focus on screening of extended family members rather than screening the general population. We have done a year wise comparison and observed that there is no change in frequency of beta thalassemia and other hemoglobinopathies (Figure 2). Even it is almost more or less from our previous studies.

Table 2. Hematological Parameters in different hemoglobinopathies

\begin{tabular}{lrlll}
\hline Category & $\mathrm{Hb}(\mathrm{g} / \mathrm{dL})$ & $\mathrm{MCV}(\mathrm{fl})$ & $\mathrm{MCH}(\mathrm{pg})$ & $\mathrm{MCHC}(\mathrm{g} / \mathrm{dL})$ \\
\hline Normal & $12.2 \pm 2.6$ & $84.5 \pm 12.7$ & $29.9 \pm 4.6$ & $36.8 \pm 2.9$ \\
$\beta \mathrm{TT}$ & $9.2 \pm 2.1$ & $69.2 \pm 6.7$ & $20.6 \pm 2.8$ & $28.6 \pm 2.2$ \\
$\beta \mathrm{TM}$ & $8.7 \pm 2.2$ & $68.7 \pm 6.0$ & $21.6 \pm 2.9$ & $25.8 \pm 4.7$ \\
$\mathrm{AS}$ & $9.1 \pm 2.4$ & $68.6 \pm 7.0$ & $21.2 \pm 3.4$ & $30.9 \pm 2.6$ \\
$\mathrm{SS}$ & $8.4 \pm 1.6$ & $69.2 \pm 7.9$ & $22.3 \pm 3.2$ & $30.3 \pm 2.4$ \\
$\mathrm{~S} \beta$ & $8.3 \pm 2.1$ & $68.6 \pm 6.2$ & $22.5 \pm 3.5$ & $31.1 \pm 2.3$ \\
$\mathrm{~S} / \mathrm{E}$ & $7.4 \pm 3.2$ & $67.1 \pm 6.9$ & $19.2 \pm 4.5$ & $28.4 \pm 3.9$ \\
$\mathrm{AE}$ & $8.6 \pm 2.4$ & $69.6 \pm 6.1$ & $21.1 \pm 3.0$ & $29.7 \pm 2.7$ \\
$\mathrm{EE}$ & $6.3 \pm 2.0$ & $64.1 \pm 6.6$ & $18.4 \pm 2.5$ & $27.7 \pm 2.5$ \\
$\mathrm{E} \beta$ & $6.0 \pm 1.4$ & $66.9 \pm 4.1$ & $18.9 \pm 2.6$ & $26.8 \pm 3.3$ \\
$\mathrm{SD}$ & $7.9 \pm 1.7$ & $91.3 \pm 0.2$ & $32.8 \pm 0.6$ & $34.2 \pm 4.9$ \\
$\mathrm{AD}$ & $10.4 \pm 2.7$ & $69.5 \pm 9.7$ & $24.0 \pm 4.8$ & $31.2 \pm 3.4$ \\
$\mathrm{DD}$ & $8.9 \pm 3.0$ & $76.1 \pm 12.7$ & $23.7 \pm 5.6$ & $31.2 \pm 1.6$ \\
\hline
\end{tabular}

Table 5 summarizes the various studies conducted in last ten years which have used the patient's selection criterion similar to our study. We can clearly see from the table that the frequency of thalassemia and other hemoglobinopathies varies significantly from one geographical region to another. The figures of World Health Organization (WHO) estimate that approximately $5 \%$ of the world's populations are carriers for genetic hemoglobin disorders. Highest (18.1\%) prevalence of beta thalassemia trait was observed in New Delhi (11) whereas it was found lowest in Dibrugarh, Assam where the HBE was more prevalent (7). Sickle cell trait, which was found in high frequency $(9.2 \%)$ in Maharashtra was found only in $0.8 \%$ of studied subjects in the present study. In our other previous studies HBS and HBE was observed in low frequency (1).

Colah et al. reported that about $1.5 \%$ of the world population are carriers of $\beta$ thalassemia (12). In central India the prevalence of $\beta$ thalassemia trait has been estimated to be $9.59 \%$ (13). In the present study the most common $\mathrm{Hb}$ abnormality detected was $\beta$ thalassemia trait present in $1185(11.0 \%)$ patients. These data reveal that for the most part of India $\beta$ thalassemia trait is a common $\mathrm{Hb}$ disorder. In this study hematological parameters were used to distinguish cases of $\beta$ thalassemia trait

Table 3. Comparison of percentage of Hemoglobin variants

\begin{tabular}{|c|c|c|c|c|c|}
\hline Category & $\mathrm{HbA}$ & $\mathrm{HbA} 2$ & $\mathrm{HbF}$ & $\mathrm{HbD}$ & $\mathrm{HbS}$ \\
\hline NORMAL & $80.5 \pm 6.9$ & $2.4 \pm 0.5$ & $0.8 \pm 0.3$ & - & - \\
\hline$\beta$ TT & $89.5 \pm 2.6$ & $5.5 \pm 0.7$ & $0.6 \pm 0.4$ & - & - \\
\hline$\beta$ TM & $24.6 \pm 28.8$ & $3.6 \pm 1.3$ & $64.1 \pm 28.4$ & - & - \\
\hline AS & $55.9 \pm 5.9$ & $3.4 \pm 0.8$ & $2.1 \pm 2.3$ & - & $34.0 \pm 11.7$ \\
\hline SS & $3.8 \pm 1.7$ & $3.4 \pm 0.7$ & $19.0 \pm 6.0$ & - & $70.1 \pm 8.4$ \\
\hline $\mathrm{S} \beta$ & $3.5 \pm 1.7$ & $5.5 \pm 0.6$ & $16.9 \pm 8.2$ & - & $72.8 \pm 8.8$ \\
\hline $\mathrm{S} / \mathrm{E}$ & $2.5 \pm 0.2$ & $49.8 \pm 12.6^{*}$ & $4.4 \pm 0.5$ & - & $43.7 \pm 13.7$ \\
\hline $\mathrm{AE}$ & $48.5 \pm 7.1$ & $33.5 \pm 11.3^{*}$ & $2.6 \pm 7.1$ & - & - \\
\hline $\mathrm{EE}$ & $5.7 \pm 2.0$ & $65.4 \pm 6.6^{*}$ & $22.8 \pm 5.9$ & - & - \\
\hline $\mathrm{E} \beta$ & $4.4 \pm 1.4$ & $64.3 \pm 14.4^{*}$ & $24.1 \pm 8.8$ & - & - \\
\hline SD & $2.6 \pm 0.1$ & $2.3 \pm 0.7$ & $16.1 \pm 5.7$ & $33.2 \pm 9.3$ & $26.3 \pm 12.7$ \\
\hline $\mathrm{AD}$ & $56.6 \pm 7.8$ & $2.3 \pm 0.6$ & $2.5 \pm 5.3$ & $33.4 \pm 5.2$ & - \\
\hline DD & $5.8 \pm 2.3$ & $3.0 \pm 1.2$ & $2.4 \pm 2.5$ & $79.8 \pm 13.1$ & - \\
\hline
\end{tabular}

*HBA2+HBE.

Table 4. Year wise distribution of incidence of anemia causing disorder

\begin{tabular}{|c|c|c|c|c|c|}
\hline $\begin{array}{l}\text { Year } \\
\text { [May-Apr] }\end{array}$ & No of Samples screened & Normal & $\begin{array}{l}\text { Thalassemia and other } \\
\text { hemoglobinopathies }\end{array}$ & IDA & Other* \\
\hline 2009-2010 & 1,010 & $203(20 \%)$ & $197(19.5 \%)$ & $590(58.5 \%)$ & $20(2 \%)$ \\
\hline 2010-2011 & 1,274 & $300(23.5 \%)$ & $210(16.4 \%)$ & $729(57.2 \%)$ & $35(2.7 \%)$ \\
\hline 2011-2012 & 1,471 & $470(31.9 \%)$ & $237(16.1 \%)$ & $728(49.4 \%)$ & $36(2.4 \%)$ \\
\hline $2012-2013$ & 1,527 & $358(23.4 \%)$ & $229(14.9 \%)$ & $899(58.8 \%)$ & $41(2.6 \%)$ \\
\hline 2013-2014 & 2,058 & $454(22.0 \%)$ & $282(13.7 \%)$ & $1,279(62.1 \%)$ & $43(2.1 \%)$ \\
\hline 2014-2015 & 2,128 & $364(17.1 \%)$ & $291(13.6 \%)$ & $1,444(67.8 \%)$ & $29(1.4 \%)$ \\
\hline 2015-2016 & 2,356 & $415(17.6 \%)$ & $351(14.8 \%)$ & $1,554(65.9 \%)$ & $36(1.5 \%)$ \\
\hline 2016-2017 & 2,542 & $368(14.5 \%)$ & $373(14.6 \%)$ & $1,745(68.6 \%)$ & $56(2.2 \%)$ \\
\hline 2017-2018 & 2,681 & $443(16.5 \%)$ & $515(19.2 \%)$ & $1,674(62.4 \%)$ & $49(1.8 \%)$ \\
\hline TOTAL, 2009-2018 & 17,047 & $3,375(19.8 \%)$ & $2,685(15.8 \%)$ & $10,642(62.4 \%)$ & $345(2.0 \%)$ \\
\hline Chi Square for trend $p$ value & & $<0.0001$ & 0.0826 & $<0.0001$ & 0.038 \\
\hline
\end{tabular}

() number in parenthesis represents percentage. 


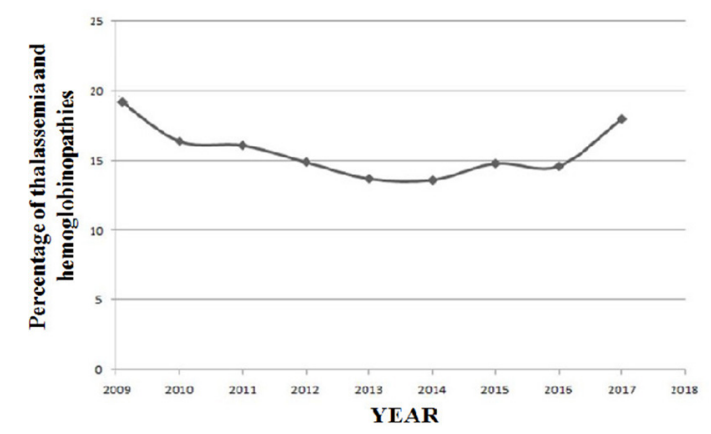

Figure 2. Year wise prevalance of thalassemia and hemoglobinopathies.

from iron deficiency anemia but these parameters were not useful in patients who have both iron deficiency and thalassemia trait. The patients with iron deficiency anemia showed significantly lower levels of $\mathrm{HbA} 2$ and MCV than the patients with a normal iron profile. In this study the rate of occurrence of iron deficiency anemia cases increases by year. There is a significant correlation with serum iron level and HbA2 concentration (14) and false diagnosis may occur in patients with iron deficiency anemia and borderline $\mathrm{HbA} 2$ (3.5-3.9\%). Other causes of borderline $\mathrm{HbA} 2$ include $\beta$ thalassemia trait with silent mutation and $\alpha$ triplication (15). So it has been emphasized that interpretation of chromatograms must be done after taking into consideration the clinical history, family history, finding of peripheral blood smear and mutation analysis for accurate diagnosis.

Several countries (Italy, Greece, Cyprus, UK, France, Iran, Thailand, Australia, Singapore, Taiwan, Hong Kong, and Cuba) prevent the birth of a thalassemia child up to $70 \%$ by setting up comprehensive national prevention programs, which include public awareness and education, carrier screening, and counseling, as well as information on prenatal diagnosis and preimplantation diagnosis (16-19). In our country also prevention programs are currently being done but they are limited to certain urban regions and these programs are done by a handful of tertiary care centers. The biggest problem in prevention of thalassemia in India is our traditions for which our society is restricted to an endogamy pattern of marriage and there is some type of social stigma that prevents them from doing thalassemia screening.

This study provided a detailed prevalence and spectrum of hereditary anemia among the North Indian population and will contribute toward the development of prevention strategies and reduction of excessive health care costs in this area, allowing better management of hemoglobinopathies. Also, there is need of a national level prevention program, which registers the thalassemia child, and sets up a national level campaign for public awareness of thalassemia and other hemoglobinopathies.

\section{Conclusion}

The present study provides a broad overview of the burden

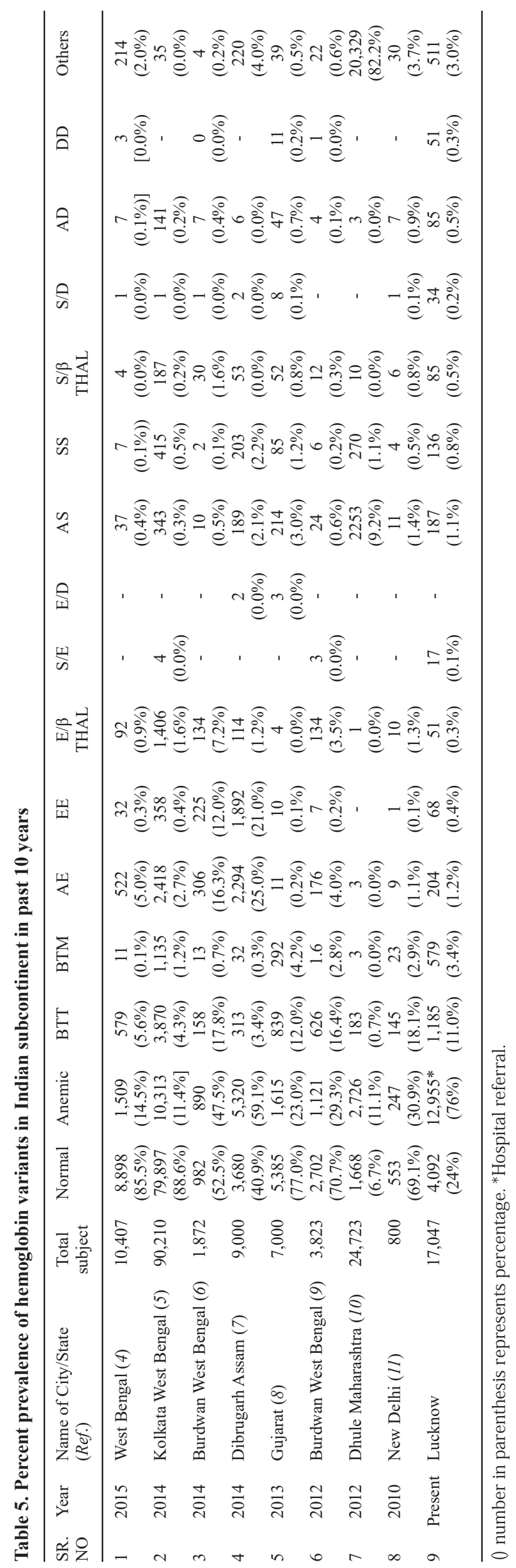


and spectrum of hemoglobinopathies in this region. The size of affected and carriers of hemoglobinopathies in the Indian population is very large. As HPLC is a powerful diagnostic tool for early detection and management of hemoglobinopathies, only a few inconclusive and unidentified cases with coexisting nutritional deficiency require further molecular analysis and iron study. Despite doing a national level screening program, extended member screening for thalassemia, antenatal screening and genetic counseling, we have seen that there is no decline in the birth of thalassemia major in India from the past.

As the centers managing hemoglobinopathies in the high prevalence area increase their experience, more and more families with this condition will come forward and cascade screening and counseling of these families will provide additional cases and carriers, and this will also provide a newer area for research. Therefore, funding by government agencies should be liberal and small scale thalassemia screening industries like laboratories should be established at various states and these centers should be linked to tertiary care hospitals so that mutational and prenatal diagnosis could be treated as prevention programs. In conclusion, a national level awareness program should be performed to prevent the birth of a thalassemia child.

\section{Acknowledgements}

The authors are thankful to SGPGIMS, Lucknow for providing infrastructure for detecting various genetic disorders. Authors are also thankful to Prof Shubha Phadke Department of Medical Genetics and Prof Sonia Nityanand Department of Hematology and their resident team for refereeing the anemia patients to Genetic Hematology lab, Medical Genetics, SGPGI, Lucknow for detailed hematological studies.

Informed consent: Informed consent was obtained from all individual participants included in the study.

\section{References}

1. Tamhankar PM, Agarwal S, Arya V, Kumar R, Gupta UR, Agarwal SS. Prevention of homozygous beta thalassemia by premarital screening and prenatal diagnosis in India. Prenat Diagn. 2009; 29:83-88.

2. Mohanty D, Colah RB, Gorakshakar AC, et al. Prevalence of $\beta$-thalassemia and other haemoglobinopathies in six cities in India: A multicentre study. J Community Genet. 2013; 4:33-42.

3. Tripathi P, Muthuswamy S, Agarwal S. A novel single nucleotide deletion mutation in exon 2 (HBB: c240delC) of beta globin gene: A case report. Int J Lab Hematol. 2017; 39:e137-e139.

4. Mukhopadhyay D, Saha K, Sengupta M, Mitra S, Datta C, Mitra PK. Role of discrimination indices in screening of beta-thalassemia trait in West Bengal, India: An institutional experience on 10,407 subjects. Saudi Journal for Health Health Sciences. 2015; 4:151-155.
5. Mondal SK, Dasgupta S, Mondal S, Das N. Spectrum of thalassemias and hemoglobinopathies in West Bengal: A study of 90,210 cases by cation exchange high performance liquid chromatography method over a period of 8 years. Journal of Applied Hematology. 2014; 5:91-95.

6. Goswami BK, Pramanik R, Chakrabarty S, Pal PP, Banerjee S, Bandyopadhyay A. Spectrum of hemoglobin variants in the population of northern region of west bengal: An ethnogenetic proposition. J Family Med Prim Care. 2014; 3:219-223.

7. Baruah MK, Saikia M, Baruah A. Pattern of hemoglobinopathies and thalassemias in upper Assam region of North Eastern India: High performance liquid chromatography studies in 9000 patients. Indian J Pathol Microbiol. 2014; 57:236-243.

8. Shrivastav A, Patel U, Joshi JR, Kaur A, Agnihotri AS. Study of hemoglobinopathies and $\mathrm{Hb}$ variants in population of Western India using HPLC: A report of 7,000 cases. Journal of Applied Hematology. 2013; 4:104-109.

9. Jain BB, Roy RN, Ghosh S, Ghosh T, Banerjee U, Bhattacharya SK. Screening for thalassemia and other hemoglobinopathies in a tertiary care hospital of West Bengal: Implications for population screening. Indian J Public Health. 2012; 56:297-300.

10. Vasaikar M, Kanthikar S, Chavan S. Spectrum of hemoglobinopathies diagnosed by HPLC in high prevalence area of north Maharashtra. International Journal of Pharma and Bio Sciences. 2012; 3:B690-697.

11. Rao S, Kar R, Gupta SK, Chopra A, Saxena R. Spectrum of haemoglobinopathies diagnosed by cation exchangeHPLC \& modulating effects of nutritional deficiency anaemias from north India. Indian J Med Res. 2010; 132:513-519.

12. Colah R, Gorakshakar A, Nadkarni A. Global burden, distribution and prevention of $\beta$-thalassemias and hemoglobin E disorders. Expert Rev Hematol. 2010; 3:103-117.

13. Chatterjee N, Mishra A, Soni R, Kulkarni H, Mamtani M, Shrivasatava M. Bayesian estimates of the prevalence of $\beta$-thalassemia trait in voluntary blood donors of central India: A survey.Hemoglobin. 2010; 34:548-560.

14. Steiner J, Marti HR, Dean D. Decreased hemoglobin A2 concentration in iron deficiency anemia. Acta Haematol. 1971; 45:77-81.

15. Giambona A, Passarello C, Vinciguerra M, Li Muli R, Teresi P, Anzà M, Ruggeri G, Renda D, Maggio A. Significance of borderline hemoglobin A2 values in an Italian population with a high prevalence of $\beta$-thalassemia. Haematologica. 2008; 93:1380-1384.

16. Hashemieh M, Timori Naghadeh H, Tabrizi Namini M, Neamatzadeh H, Hadipour Dehshal M. The Iran thalassemia prevention program: Success or failure? Iran J Ped Hematol Oncol. 2015; 5:161-166.

17. Cao A, Kan YW. The prevention of thalassemia. Cold Spring Harb Perspect Med. 2013; 3:a011775.

18. Khorasani G, Kosaryan M, Vahidshahi K, Shakeri S, Nasehi MM. Results of the national program for prevention of beta-thalassemia major in the Iranian Province of Mazandaran. Hemoglobin. 2008; 32:263-271.

19. Bozkurt G. Results from the north cyprus thalassemia prevention program. Hemoglobin. 2007; 31:257-264.

(Received August 30, 2018; Revised November 18, 2018; Accepted November 23, 2018) 